\title{
Weyl groups of small groups of finite Morley rank
}

\author{
Jeffrey Burdges* and Adrien Deloro
}

November 7, 2007

\section{Introduction}

Infinite groups of finite Morely rank have little truly geometric structure; however, their algebraic properties are remarkably reminiscent of algebraic groups. The strongest conjecture to this effect is the Cherlin-Zil'ber algebraicity conjecture which postulates that an infinite simple group of finite Morley rank is a linear algebraic group over an algebraically closed field.

There are a number of partial results towards this conjecture and a complete proof in the even $\mathscr{E}$ mixed type cases, read potentially characteristic two. In other cases, much recent work has followed two themes : an analysis of the minimal simple groups where Bender's method is well understood [Bur07], and the analysis of torsion using genericity arguments [BBC07, BC08], as well as work involving both techniques [BCJ07, Del07, AB08].

A major part of the combined thread is the analysis of the Weyl group $W:=N(T) / C^{\circ}(T)$ of $G$ associated to some maximal decent torus $T$. Here a decent torus is merely the smallest definable subgroup containing some divisible abelian torsion subgroup, such as a $p$-torus $\mathbb{Z}\left(p^{\infty}\right)^{n}$. In fact the reader my may always replace decent torus by $p$-torus. One may speak of the Weyl group of $G$ because maximal tori are conjugate in a group of finite Morley rank [Che05].

In the present article, we show that the Weyl group is cyclic in a minimal connected simple group of finite Morley rank.

Theorem 3.1. Let $G$ be a minimal connected simple group of finite Morley rank, and let $T$ be a decent torus of $G$. Then the Weyl group $W:=N(T) / C(T)$ is cyclic.

We also give a condition on the Prüfer $p$-rank of a torus that admits $p$ as a divisor of $|W|$. The Prüfer $p$-rank $\operatorname{pr}_{p}(T)$ is merely the maximal $n$ such that $\mathbb{Z}\left(p^{\infty}\right)^{n}$ is a subgroup of $T$.

Theorem 4.1. Let $G$ be a minimal connected simple group of finite Morley rank, and let $T$ be a nontrivial p-torus of $G$. Suppose that $p$ is a prime divisor of $\left|W_{T}\right|$. Then $p-1$ divides the Prüfer $p$-rank $\operatorname{pr}_{p}(T)$ of $T$, and $p$ is not the minimal prime divisor of $|W|$ unless $p=2$.

*Supported by NSF postdoctoral fellowship DMS-0503036. 
Our primary concern here is groups of degenerate type, where Sylow 2subgroups are trivial [BBC07]. The second authors thesis work covers the odd type case (see Fact 3.2 below) and the afore mentioned classification in even \& mixed types covers those cases.

As an introduction, we first prove a far stronger yet easier result, but under strong number theoretic hypotheses.

\section{Tameness Wagner style}

The Weyl group of an algebraic group is the quotient of the normalizer of a maximal algebraic torus by the centralizer of the same torus. In general, groups of finite Morley rank need not contain any algebraic torus, but frequently have so-called decent tori. A decent torus is a divisible abelian group which is the definable hull of its torsion. The group $W_{T}:=N(T) / C(T)$ is the Weyl group associated to a given torus $T$. Here one may use only the connected component of the centralizer by the following.

Fact 2.1 ([AB08, Theorem 1]). Let $T$ be a decent torus of a connected group $H$ of finite Morley rank, Then $C(T)$ is connected.

We naturally say "the Weyl group of $G$ " when $T$ is a maximal decent torus; this is well defined by conjugacy of maximal decent tori [Che05].

Lemma 2.2. Let $G$ be a minimal connected simple group of finite Morley rank, and let $T$ be a nontrivial decent torus of $G$. Then the Weyl group $W_{T}:=$ $N(T) / C(T)$ associated to $T$ is naturally isomorphic to a subgroup of the Weyl group $W$ of $G$.

Here we'll use the fact that solvable groups have trivial Weyl groups (see also [AB08, Lemma 5.11]).

Fact 2.3 ([AB08, Lemma 6.6]). Let $H$ be a connected solvable group of finite Morley rank and let $K$ be a definable connected subgroup of $H$ such that $\left[N_{H}(K): K\right]<\infty$. Then $N_{H}(K)=K$.

Proof of Lemma 2.2. Let $S$ be a maximal decent torus containing $T$. Then $S \leq$ $C^{\circ}(T) \triangleleft N(T)$. So $W_{T}$ is a section of $W:=N(T) / C(T)$ by a Frattini argument using [Che05]. Of course the kernel here is the Weyl group $N_{C(T)}(S) / C_{C(T)}(S)$ of $S$ inside $C(T)$. But $C(T)$ is solvable by Fact 2.1. So this kernel is trivial by Fact 2.3, as desired.

A similar argument shows that, in a minimal connected simple group, the Weyl group is isomorphic to $W_{Q}:=N(Q) / Q$ where $Q$ is a Carter subgroup containing the maximal torus $S$. In a minimal simple group, one need not specify the Carter subgroup $Q$ as they are all conjugate [Fré08] but this need not hold in general.

In the present article we examine some variations on the following result. 
Theorem 2.4. Let $G$ be a minimal connected simple group of finite Morley rank and degenerate type, and let $T$ be a nontrivial decent torus of $G$. Then either

1. the Weyl group $W:=N(T) / C(T)$ is trivial, or

2. $G$ interprets a bad field in characteristic $p$ where $p$ is at most the minimal prime divisor of $|W|$.

Here a bad field $(k, H,+, \cdot)$ is a field $k$ of finite Morley rank with a proper nontrivial definable subgroup $H$ of it's multiplicative subgroup. Such fields exist in characteristic zero but are quite unlikely in positive characteristic $p$ for number theoretic reasons. In this second situation, there are only finite many primes of the form $\frac{p^{n}-1}{p-1}$ by [Wag97]. Moreover the asymptotics in [HW] hold :

$$
\left(p^{n}-1\right)_{\pi} \asymp p^{\alpha n}
$$

Here $\pi$ denotes the set of primes $\pi$ appearing in the bad field's multiplicative subgroup, and $\alpha$ denoted the ratio $\operatorname{rk}(T)$ So our theorem says that Weyl groups are unlikely degenerate type.

To prove the theorem, we consider a minimal connected simple group $G$, and a nontrivial decent torus $T$ of $G$. By Lemma 2.2, we may assume that $T$ is a maximal decent torus.

Let $p$ be the minimal prime divisor of $W:=N(T) / C(T)$. Then $G$ contains $p$-unipotence by the following important fact.

Fact 2.5 ([BC08, Theorem 5]). Let $H$ be a connected group of finite Morley rank. Suppose the Weyl group associated to a maximal decent torus is nontrivial and has odd order, with $p$ the smallest prime divisor of its order. Then $H$ contains a unipotent p-subgroup.

Hence there is a Borel subgroup $B$ of $G$ with $U_{p}(B) \neq 1$. So consider some $B$-minimal $A \leq U_{p}(B)$.

Suppose first that $C_{B}(A)<B$. By the Zilber field theorem, there is a field $k$ interpretable in $B$ with $A \cong k_{+}$and $B / C_{B}(A) \hookrightarrow k^{*}$. If $G$ has degenerate type, $k$ is our bad field in characteristic $p$, as desired.

So suppose otherwise that $C_{B}(A)=B$. Then $B \cap B^{g}=1$ for $g \notin N(B)$ by the Jaligot Lemma :

Fact 2.6 ([Bur07, Lemma 2.1]). Let $B_{1}, B_{2}$ be two distinct Borel subgroups of $G$ satisfying $U_{p_{i}}\left(B_{i}\right) \neq 1$ for some prime $p_{i}(i=1,2)$. Then $F\left(B_{1}\right) \cap F\left(B_{2}\right)=1$.

It follows that $\bigcup B^{G}$ is generic in $G$ by the genericity argument :

Fact 2.7 ([BBC07, Lemma 4.1]). Let $G$ be a group of finite Morley rank, $H$ a definable subgroup of $G$, and $X$ a definable subset of $G$. Then $\operatorname{rk}\left(\bigcup X^{G}\right)=\operatorname{rk}(G)$ whenever

$$
\operatorname{rk}\left(X \backslash \bigcup_{g \notin H} X^{g}\right) \geq \operatorname{rk}(H) .
$$


But now $B$ contains a maximal decent torus of $G$ by the following.

Fact 2.8 ([BC08, Theorem 1]). Let $G$ be a connected group of finite Morley rank and $a$ be a generic element of $G$. Then $d(a)$ contains a maximal decent torus of $G$.

So we may assume that $B=C^{\circ}(T)$, and $p$ divides $N(B) / B$, contradicting the following.

Fact 2.9 ([AB08, Lemma 4.3]). Let $B$ be a Borel subgroup of $G$ such that $U_{p}(B) \neq 1$ for some prime $p$. Then $p \nmid\left[N_{G}(B): B\right]$.

This concludes the proof of Theorem 2.4.

\section{Cyclicity of Weyl groups}

Theorem 2.4 completes our picture of Weyl groups in minimal connected simple groups of degenerate type, if there are no bad fields in positive characteristic. However, one can still prove something without such strong hypotheses.

Theorem 3.1. Let $G$ be a minimal connected simple group of finite Morley rank, and let $T$ be a decent torus of $G$. Then the Weyl group $W_{T}:=N(T) / C(T)$ is cyclic.

We may start the proof with a non-trivial decent torus $T$ which by Lemma 2.2 can be taken maximal. Again recall that $C(T)$ is connected by Fact 2.1.

We dispense with the odd type case by using the second author's thesis work. As observed after the proof of Lemma 2.2, letting $Q$ be a Carter subgroup of $G$ containing $T$, we find $W \cong W_{Q}:=N(Q) / Q$.

Fact 3.2 ([Del07, see Théorème p.89]). Let $G$ be a minimal connected simple group of finite Morley rank of odd type, and let $Q$ be a Carter subgroup of $G$ containing a Sylow 2-subgroup. Then $|N(Q) / Q|=1,2,3$.

So $|W|=1,2,3$ if such a $G$ has odd type. We may therefore assume that $G$ has degenerate type by the Even Type Theorem [ABC07].

The real starting point for our analysis is an earlier result about Weyl groups in minimal connected simple groups.

Fact 3.3 ([AB08, Proposition 5.1 \& Corollary 5.7]). $W$ is a metacyclic Frobenius complement.

A Frobenius complement is the stabilizer of a point in a Frobenius group; whch is a transitive permutation group on a finite set, such that no non-trivial element fixes more than one point and some non-trivial element fixes a point. Such groups have a quite restrictive structure, which is described by the following.

Fact 3.4 ([Gor80, 10.3 .1 p. 339]). Let $W$ be a Frobenius complement. Then 
1. Any subgroup of $W$ of order $p q, p$ and $q$ primes, is cyclic.

2. Sylow p-subgroups of $W$ are either cyclic or possibly generalized quaternion if $p=2$.

Of course metacyclicity follows for any Frobenius complement inside a degenerate type group [Gor80, 7.6.2 p. 258].

Consider a minimal non-cyclic subgroup $\Xi$ of $W$. Then $\Xi$ is metacyclic but not cyclic while every proper subgroup of $\Xi$ is cyclic. In particular every proper subgroup of $\Xi$ is abelian yet $\Xi$ itself is not.

Claim 3.5. There are prime numbers $p$ and $q \mid p-1$ and elements $\alpha, \beta \in \Xi$ with $\alpha^{p}=1, \beta^{q^{m}}=1$, and $\beta^{q} \neq 1$ such that :

- $\Xi=\langle\alpha, \beta\rangle$;

- $\left[\alpha, \beta^{q}\right]=1$;

- $\Xi^{\prime}=\langle\alpha\rangle$.

Proof. Say $\Xi / C_{1} \cong C_{2}$ where $C_{1}$ and $C_{2}$ are cyclic groups. Let $\alpha \in \Xi$ be a generator of $C_{1}$, and let $\beta$ be an element of $\Xi$ whose image modulo $C_{1}$ generates $C_{2}$. Of course $[\alpha, \beta] \neq 1$ since $\Xi$ is not abelian.

By minimality of $\Xi, \alpha$ and $\beta$ must have prime powers as orders, say $p^{n}$ and $q^{m}$ respectively. It follows that $p \neq q$ because Sylow $p$-subgroups are cyclic.

Also notice that by minimality:

$$
\left[\alpha^{p}, \beta\right]=\left[\alpha, \beta^{q}\right]=1
$$

So $\langle\beta\rangle \simeq \mathbb{Z} / q^{m} \mathbb{Z}$ normalizes $C_{1}=\langle\alpha\rangle \simeq \mathbb{Z} / p^{n} \mathbb{Z}$. As $X$ is non-abelian, the action is non-trivial. Recall that any group automorphism of $\mathbb{Z} / p^{n} \mathbb{Z}$ as a group is also a ring automorphism. Our action of $\beta$ on $\langle\alpha\rangle$ by conjugation therefore becomes a multiplicative action on $\mathbb{Z} / p^{n} \mathbb{Z}$ by some $1 \neq \lambda \in \mathbb{Z} / p^{n} \mathbb{Z}$.

By $(\dagger)$, the action of $\lambda^{q}$ is trivial, i.e. $\lambda^{q} \equiv 1\left(\bmod p^{n}\right)$. On the other hand, $b$ acts trivially on $\left\langle\bar{a}^{p}\right\rangle$, so $p \lambda \equiv p\left(\bmod p^{n}\right)$. This means $p^{n-1} \mid \lambda-1$. Hence there is an integer $k \in\{1, \ldots, p-1\}$ such that $\lambda \equiv 1+k p^{n-1}\left(\bmod p^{n}\right)$. Raising to the $q$ th power, we find $1 \equiv \lambda^{q} \equiv\left(1+k p^{n-1}\right)^{q}\left(\bmod p^{n}\right)$.

Now, if $n$ were $\geq 2$, we'd have $1 \equiv 1+q k p^{n-1}\left(\bmod p^{n}\right)$ because all other terms are divisible by at least $p^{2(n-1)}$, which is trivial in $\mathbb{Z} / p^{n} \mathbb{Z}$. So, if $n \geq 2$, we derive $p \mid q k$, which is absurd. Therefore $n=1$, and $\alpha$ has order exactly $p$.

Now Fact 3.4(1) and non-abelianness of $\Xi$ together imply that $\beta^{q}$ must be non-trivial.

Clearly $\langle\alpha\rangle$ contains $\Xi^{\prime} \neq 1$. As $[\alpha, \beta] \neq 1$, everything is proved.

Our next step is to lift $\Xi$ isomorphically to a subgroup $X \leq N(T)$, which will require some attention.

Let $a$ be a $p$-element of $N(T) \backslash C^{\circ}(T)$ whose image in $\Xi \leq W$ is $\alpha .{ }^{1}$ Such liftings exist by the usual torsion lifting principle [BN94, Ex. 11 p. 93; Ex. 13c p. 72]. However, such a wild lifting of $b$ need not normalize our $\langle a\rangle$.

\footnotetext{
${ }^{1}$ Let's be consistent about the use of notation blocks.
} 
Claim 3.6. $C(T)$ has $p^{\perp}$-type.

Proof. Otherwise we suppose towards a contradiction that $U_{p}(C(T)) \neq 1$. Then $C(T)$ lies inside a unique Borel subgroup $B$ by Fact 2.6. In particular $B$ is normalized by the Weyl element representative $a$. As $U_{p}(B) \neq 1$, it follows that $a \in B$ using Fact 2.9, but this contradicts Fact 2.3.

In consequence, [BC08, Theorem 4] says that any definable subgroup of $N(T)$ satisfies conjugacy of Sylow $p$-subgroups

We let $T_{p}$ denote the maximal $p$-torus in $T$, which might be trivial.

Claim 3.7. There is a q-element $b_{0}$ lifting $\beta$ and normalizing $\langle a\rangle T_{p}$.

Proof. Let $\pi: N(T) \rightarrow N(T) / C(T)$ denote the quotient map, and let $H=$ $\pi^{-1}(\Xi)$ and $K=\pi^{-1}(\langle\alpha\rangle)$. Of course $K \triangleleft H$ by Claim 3.5. Also, $\langle a\rangle T_{p}$ is clearly a Sylow $p$-subgroup of $K$. As Sylow $p$-subgroups of $K$ are $K$-conjugate, a Frattini argument yields $H=K \cdot N_{H}\left(\langle a\rangle T_{p}\right)$. So $N\left(\langle a\rangle T_{p}\right)$ meets the coset $\beta$. Again torsion lifting yields a $q$-element $b_{0}$ in $N\left(\langle a\rangle T_{p}\right)$ whose image is $\beta$.

Claim 3.8. The group $X_{0}:=\left\langle a, b_{0}\right\rangle$ is finite.

Proof. As $\langle a\rangle T_{p}$ is locally finite, so is $\left(\langle a\rangle T_{p}\right) \cdot\left\langle b_{0}\right\rangle \geq\left\langle a, b_{0}\right\rangle$.

Our $X_{0}$ needn't yet be isomorphic to $\Xi$; however, it's elements retain their original orders.

Lemma 3.9. Let $G$ be a minimal connected simple group of finite Morley rank, let $T$ be a nontrivial maximal torus, and let $\bar{x} \in N(T) / C(T)$. Then any lifting of $\bar{x}$ to a torsion element $x \in N(T) \backslash C(T)$ has order $|\bar{x}|$. In other words $\langle x\rangle \cap C(T)=1$ whenever $x \in N(T) \backslash C(T)$ is torsion.

Here we use the that minimal simple groups are covered by their Borel subgroups.

Fact 3.10 ([AB08, Corollary 4.4]). Let $G$ be a minimal connected simple group of finite Morley rank. Any torsion element $x$ of $G$ lies inside any Borel subgroup of $G$ which contains $C^{\circ}(x)$.

Proof of Lemma 3.9. Consider a lifting $x$ of finite order with $x^{n} \in C(T)$. Suppose towards a contradiction that $x^{n}=1$. Fix some Borel subgroup $B$ contain$\operatorname{ing} C^{\circ}\left(x^{n}\right) \geq C^{\circ}(x)$. Since $x$ is torsion, $x \in B$ by Fact 3.10. But $T \leq C^{\circ}\left(x^{n}\right) \leq$ $B$ too, contradicting Fact 2.3.

In particular $a$ has order $p$ and $b_{0}$ order $q^{m}$.

To prove the isomorphism, we require the following observation.

Lemma 3.11. Let $T$ be a torus in a minimal connected simple group, and let $x \in N(T) \backslash C(T)$. Then the function $\varphi: T \rightarrow$ given by $t \mapsto[t, x]$ is a surjective endomorphism. 
Proof. The map $\varphi$ is obviously a group homomorphism. Now $\operatorname{ker} \varphi=C_{T}(x)$. If the latter is infinite, then there is a non-trivial subtorus $1 \neq \tau \leq T$ such that $x \in C(\tau)=C^{\circ}(\tau)$ by Fact 2.1. Hence $C^{\circ}(\tau)$ contains the group $T$. $\langle x\rangle$, a contradiction to Fact 2.3. This proves that $C_{T}(x)$ is finite, hence $\varphi$ is surjective.

Claim 3.12. There is a q-element $b$ lifting $\beta$ and normalizing $\langle a\rangle$.

Proof. A translation $t b_{0}$ of $b_{0}$ by some $t \in T$ has the same $q^{m}$, by Lemma 3.9. So we search for a $t$ for which $b$ normalizes $\langle a\rangle$.

Since $\beta$ normalizes $\langle\alpha\rangle$ but does not centralize it, there is an integer $1<k<p$ with $\alpha^{\beta}=\alpha^{k}$. So there is an element $s \in C(T)$ such that $a^{b_{0}}=s a^{k}$, and clearly $s \in X_{0}$ has finite order. Since $b_{0}$ normalizes $\geq a T_{p}$ and $a$ normalizes $T_{p}$, we actually have $s \in T_{p} \leq T$.

By Lemma 3.11, there is a $t \in T$ be such that $\left[t, a^{-1}\right]=s^{-b_{0}-1}$. Set $b:=t b_{0}$. Then still $\pi(b)=\beta$. As $b^{q^{m}} \in T$ has finite order, $b$ has order $q^{m}$ by Lemma 3.9. Furthermore,

$$
a^{b}=\left(a^{t}\right)^{b_{0}}=\left(\left[t, a^{-1}\right] a\right)^{b_{0}}=\left[t, a^{-1}\right]^{b_{0}} a^{b_{0}}=s^{-1} s a^{k} .
$$

So now $X:=\langle a, b\rangle \leq N(T)$ is an isomorphic group lifting $\Xi$.

We next push our subgroup $X$ into some Borel subgroup of $G$, after first extracting one more consequence of Borel covering (Fact 3.10).

Lemma 3.13. Let $G$ be a minimal connected simple group of finite Morley rank. If $U$ is a finite subgroup of $G$ with a cyclic Sylow $r$-subgroup $R$ that meets $Z(U)$, then $U$ lies inside some Borel subgroup of $G$; in fact any Borel subgroup containing $C^{\circ}(z)$ for some $z \in Z(U)$.

Proof. Fix $z \in Z(U)$ of order $r$, and let $B$ be a Borel containing $C^{\circ}(z)$. Consider some $x \in U$. If $r$ divides $|x|$ then $z$ is a power of $y:=x$. If $r$ does not divide $|x|$ then $z$ is a power of $y:=x z$. In either case $C^{\circ}(y) \leq C^{\circ}(z)$. So $y \in B$ by Fact 3.10. Hence $x \in\langle y\rangle$ lies inside $B$ too.

Claim 3.14. There is a Borel subgroup $B$ of $G$ containing $X$.

Proof. Suppose first that $a^{p} \neq 1$. By Claim 3.5, we find that $Y:=\left\langle a^{p}, b\right\rangle$ is cyclic, and $a^{p}$ is central in $X$. So $B$ exists by Lemma 3.13 .

Suppose alternatively that $a^{p}=1$. Then, since $[a, b] \neq 1$, one has $b^{q} \neq 1$ by Fact 3.4 (i). Again $Y:=\left\langle a, b^{q}\right\rangle$ is cyclic by Claim 3.5, and now $p^{q}$ is central in $X$. So once again $B$ exists by Lemma 3.13 .

Of course $X$ determines the roles of $a$ and $b$ inside $B$.

Claim 3.15. $X^{\prime}=\langle a\rangle \leq U_{p}(B)$ and $b \notin F(B)$ 
Proof. Let $S_{p}$ be a Sylow $p$-subgroup of $B^{\prime}$ containing $a$. By [BN94, Theorem $9.29 \&$ Corollary 6.20], $S_{p}=U_{p}(B) * T_{p}$ where $T_{p}$ is some maximal $p$-torus of $F(B)$. Of course $T_{p}$ is central in $B$ by [BN94, Theorem 6.16]. So $a \notin T_{p}$ since $[a, b] \neq 1$. The cylic group $X^{\prime}=\langle a\rangle$ is the Sylow $p$-subgroup of $X$. So $a \in U_{p}(B)$.

Also $b \notin F(B)$ since $X$ is non-nilpotent.

We now find an honestly toral alternative to $b$ who still centralizes some unipotent element related to $a$. In fact such an element must centralize an infinite unipotent subgroup by the following lemma.

Lemma 3.16. Let $B$ be a Borel subgroup with $U_{p}(B) \neq 1$, and $t \in B$. If $C_{U_{p}(B)}(t) \neq 1$ then it is infinite.

Here one uses the following fact about relatively prime actions.

Fact 3.17 ([ABCC01], [Bur04, Fact 3.3]). Let $H=K T$ be a group of finite Morley rank. Suppose that $T$ is a solvable $\pi$-group of bounded exponent and that $K$ is a definable abelian normal $\pi^{\perp}$-subgroup of $H$. Then $H=[H, T] \oplus C_{H}(T)$.

Proof of Lemma 3.16. Let $x \in C_{U_{p}(B)}(t)^{\#}$ and let $Z_{i}:=Z_{i}^{\circ}\left(U_{p}(B)\right)$ for $i \in \mathbb{N}$. As $U_{p}(B)$ is nilpotent and connected, there is some integer $i$ such that $x \in$ $Z_{i+1} \backslash Z_{i}$. In particular the action of $t$ on the connected abelian quotient $Y:=$ $Z_{i+1} / Z_{i}$ has some centralization. By Fact 3.17, $C_{Y}(t) \cong Y /[Y, t]$ is connected and infinite, and hence $C_{Y}(t)$ is infinite. As now $U_{p}\left(C_{Y}(t)\right) \neq 1$, it follows that $U_{p}\left(C_{B}(t)\right) \neq 1$.

Claim 3.18. There is a toral q-element $t$ of $B$ with an infinite centralizer in either $U_{p}(B)$ or possibly $U_{q}(B)$. In particular $B$ is the only Borel containing $C_{B}^{\circ}(t)$.

Proof. Fix some Sylow $q$-subgroup $S_{q}$ of $B$ containing $b$. By [BN94, Theorem $9.29 \&$ Corollary 6.20], $S_{q}=T_{q} * U_{q}(B)$, where $T_{q}$ is a $q$-torus of $B$. We thus have $T_{q} \neq 1$ because $S_{q}$ cannot centralize $a \in U_{p}(B)$ while $U_{q}(B)$ does. So we may assume $U_{q}(B)=1$ since otherwise any element of $T_{q}$ suffices. In particular $b \in T_{q}$ is toral.

By Claims 3.5, 3.12, and 3.15, $b^{q}$ centralizes $a \in U_{p}(B)^{\#}$. So our main conclusion follows from Corollary 3.16. Now our last conclusion follows from Fact 2.6.

At this point, the structure surrounding $B$ solidifies considerably.

Fix $g \in G$ such that $t \in T^{g}$. Set $T_{1}:=T^{g}$. Of course $T_{1} \leq B$ by Claim 3.18.

Claim 3.19. There is no Borel subgroup of $G$ both containing $T$ and meeting $N(T) \backslash C^{\circ}(T)$.

Proof. Suppose there is a Borel subgroup $B_{0}$ of $G$ containing both $T$ and some $c \in N(T) \backslash C(T)$. As $B_{0}$ is connected and solvable, $N_{B_{0}}(T)=C_{B_{0}}(T)$ by Fact 2.3. So $c$ centralizes $T$, a contradiction. 
Claim 3.20. $a^{g} \notin N(B)$.

Proof. Here $a^{g} \notin B$ because $a \notin B^{g^{-1}}$ by Claim 3.19. So $a^{g} \notin N(B)$ by Fact 2.9 .

Lemma 3.21. The Prüfer $p$-rank $\operatorname{pr}_{q}\left(T_{1}\right)$ of $T_{1}$ is at least 3 .

Proof. Suppose first that $\operatorname{pr}_{q}\left(T_{1}\right)=1$. Then $a^{g} \in N\left(T_{1}\right)$ normalizes $\langle t\rangle$. As $B$ is the only Borel subgroup containing $C^{\circ}(t), a^{g}$ also normalizes $B$, in contradiction with Claim 3.20.

Suppose next that $\operatorname{pr}_{q}\left(T_{1}\right)=2$. We have $p>q+1$ because $q \mid p-1$ and $q \neq 2$. So there is no injective homomorphisms from $\mathbb{Z} / p \mathbb{Z}$ to $\mathrm{GL}_{2}(\mathbb{Z} / q \mathbb{Z})$. So $a^{g}$ must centralize $t$, again contradicting Claim 3.20.

We now derive our final contradiction. By the following, there is an elementary abelian $q$-group $E_{0} \leq E:=\Omega_{1}(T)$ with $m_{q}\left(E_{0}\right) \geq 2$ such that $C_{U_{p}(B)}\left(E_{0}\right) \neq 1$.

Fact 3.22 ([Bur04, Fact 3.7]). Let $q$ be a prime number. Let $H$ be a solvable $q^{\perp}$-group of finite Morley rank. Let $E$ be a finite elementary abelian q-group acting definably on $H$. Then

$$
H=\left\langle C_{H}\left(E_{0}\right): E_{0} \leq E,\left[E: E_{0}\right]=q\right\rangle .
$$

By Fact 3.22 again, there is a $v \in E_{0}^{\#}$ such that $C_{U_{p}\left(B^{a}\right)}(v) \neq 1$ too. But now $U_{p}\left(C^{\circ}(v)\right)$ meets both $U_{p}(B)$ and $U_{p}\left(B^{a}\right)$ nontrivially, a contradiction to Fact 2.6.

This concludes the proof of theorem 3.1.

\section{Possible Prüfer $p$-ranks}

We're aware, thanks to Fact 2.5, that a nontrivial Weyl group $W$ produces $p$ unipotence for some divisor $p$ of $|W|$; making $p$-tori seem unlikey. Here we show that such a $p$-torus has only a limited selection of the Prüfer $p$-ranks.

Theorem 4.1. Let $G$ be a minimal connected simple group of finite Morley rank, and let $T$ be a nontrivial $p$-torus of $G$. Suppose that $p$ is a prime divisor of $\left|W_{T}\right|$. Then $p-1$ divides the Prüfer $p$-rank $\operatorname{pr}_{p}(T)$ of $T$, and $p$ is not the minimal prime divisor of $|W|$ unless $p=2$.

The proof entirely relies on the following observation.

Lemma 4.2. Let $T$ be a p-torus and $\alpha$ be an automorphism of order $p$ of $T$. Then:

- T has Prüfer-rank at least $p-1$;

- if $T$ has Prüfer-rank $p$ and lives inside a group of finite Morley rank in which $\alpha$ is a definable automorphism of $T$, then $C_{T}(\alpha)$ is infinite. 
Here we apply the Tate module construction of a complex representation for our Weyl group.

Fact 4.3 ([Ber01, BB04, §3.3]). Let $T$ be a p-torus of Prüfer $p$-rank $n$. Then $\operatorname{End}(T)$ can be faithfully represented as the ring $M_{n \times n}\left(\mathbb{Z}_{p}\right)$ of $n \times n$ matrices over the p-adic integers $\mathbb{Z}_{p}$.

Proof of Lemma 4.2. Recall that $1+X+\cdots+X^{p-1}$ is irreducible in $\mathbb{Q}_{p}[X]$. In the Tate module, $\alpha$ is represented by a matrix $M \in \mathrm{GL}_{d}\left(\mathbb{Z}_{p}\right) \leq \mathrm{GL}_{d}\left(\mathbb{Q}_{p}\right)$ where $d=\operatorname{pr}_{p}(T)$. As $M^{p}=1$, the minimal polynomial $\mu$ of $M$ must divide $X^{p}-1$. Since $\alpha \neq \mathrm{Id}$, we have $M \neq \mathrm{Id}$, so $\mu \neq X-1$. Therefore $\mu \neq X-1$ divides $X^{p}-1=(X-1)\left(1+X+\cdots+X^{p-1}\right)$. As $1+X+\cdots+X^{p-1}$ is irreducible over $\mathbb{Q}_{p}$, it follows that $1+X+\cdots+X^{p-1}$ divides $\mu$.

So the minimal polynomial has degree at least $p-1$. By the Cayley-Hamilton Theorem, so must the characteristic polynomial; but the latter has degree $d$, whence $d \geq p-1$. This proves the first claim.

Also, if $d=p$, then the characteristic polynomial is $\left(1+X+\cdots+X^{p-1}\right)(X-a)$ for some element $a \in \mathbb{Q}_{p}$, which is an eigenvalue of $M$. So $a$ has multiplicative order $p$ in $\mathbb{Q}_{p}$, and this proves $a=1$. Let $x$ be an eigenvector for the eigenvalue 1. We may assume that $x$ lies in $\mathbb{Z}_{p}^{d} \backslash \mathbb{Z}^{d}$.

Now projecting, we deduce that at every stage $\left(\mathbb{Z} / p^{n} \mathbb{Z}\right)^{d}, \alpha$ centralizes an element $x_{n}$ of order $p^{n}$. By compactness, $\alpha$ centralizes some element of infinite order. Therefore $C_{T}(\alpha)$ is infinite.

We now begin the proof of Theorem 4.1. Let $T$ be a $p$-torus where $p$ divides $\left|W_{T}\right|$. Let $g$ be a $p$-element of $N(T)$ that has order $p$ in $W_{T}$, i.e. $g^{p} \in C(T)$.

Claim 4.4. $C_{T}(g)$ is finite.

Proof. If not, then $\tau=C_{T}^{\circ}(g)$ is a non-trivial subtorus of $T$. In particular, Fact 2.1 says that $C(\tau)$ is a connected solvable group. Since it contains the $p$-group $T \cdot\langle g\rangle$, we find $g \in C(T)$, in contradiction with Fact 2.3.

Let $T_{0} \leq T$ be a subtorus of Prüfer $p$-rank exactly 1 . Let $T_{1}=T_{0}+T_{0}^{g}+$ $\cdots+T_{0}^{g^{p-1}}$. This is a non-trivial $g$-invariant $p$-torus of Prüfer rank at most $p$.

Claim 4.5. $T_{1}$ has Prüfer rank $p-1$.

Proof. If either $\operatorname{pr}_{p}\left(T_{1}\right)<p-1$ or $\operatorname{pr}_{p}\left(T_{1}\right)=p$, then Lemma 4.2 says that $g$ centralizes a non-trivial subtorus of $T_{1}$. But this contradicts Claim 4.4.

Claim 4.6. There is a g-invariant subtorus $T^{\prime}<T$, possibly trivial, with Prüfer-rank $\operatorname{pr}_{p}\left(T^{\prime}\right)=\operatorname{pr}_{p}(T)-(p-1)$.

Proof. Since we're working with an automorphism of order $p$, the intersection $T_{1} \cap T^{\prime}$ may be non-trivial but remains finite. The rank computation follows as in Maschke's theorem. ${ }^{2}$

\footnotetext{
${ }^{2}$ I've not actually $100 \%$ sure about this comment anyway, change as you like.
} 
It follows, by induction on $\operatorname{pr}_{p}(T)$, that $T^{(k)}=0$ eventually. So $\operatorname{pr}_{p}(T)=$ $k(p-1)$ as desired.

This proves the first part of Theorem 4.1.

We prove the second as follows.

Proposition 4.7. Let $G$ be a minimal connected simple group of finite Morley rank, let $T$ be a decent torus of $G$, and let $a \in N(T) \backslash C(T)$ be a p-element. Then either $\operatorname{pr}_{p}(T)=0$ or $U_{p}(C(a))=1$.

Of course both are impossible together by [BC08, Theorem 3].

Proof. Otherwise suppose that both $\operatorname{pr}_{p}(T)=0$ and $U_{p}(C(a))=1$ hold. As $U_{p}(C(a)) \neq 1, C^{\circ}(a)$ lies inside a unique Borel subgroup $B_{a}$ by Fact 2.6. Let $T_{p}$ be the $p$-torus of $T$, and its nontrivial. Then there is some nontrivial $z \in$ $Z\left(\langle a\rangle T_{p}\right) \cap T_{p}$ by [BN94, ???]. So $z$ normalizes $C^{\circ}(a)$, and hence $B_{a}$. Hence $z \in B_{a}$ by Fact 2.9 , and $U_{p}\left(C_{B_{a}}(z)\right) \neq 1$ too. But $T \leq C(z)$. So $U_{p}\left(C\left(T_{p}\right)\right) \neq 1$. Again, by Fact 2.6, $C\left(T_{p}\right)$ lies inside a unique Borel subgroup $B_{z}$, and $a \in B_{z}$ by Fact 2.9. But $N_{B_{z}}(T)=C_{B_{z}}(T)$ by Fact 2.3, a contradiction.

Corollary 4.8. Let $G$ be a minimal connected simple group whose Weyl group $W$ has odd order. Then $G$ has no divisible $p$-torsion for $p$ the minimal prime divisor of $|W|$.

The corollary is a direct consequence of the following variation on Fact 2.5 above.

Fact 4.9 ([BC08, Corollary 5.3]). Let $G$ be a minimal connected simple group of finite Morley rank. Suppose the Weyl group is nontrivial and has odd order, with $r$ the smallest prime divisor of its order. Then any $r$-element representing an element of order $r$ in $W$ centralizes a unipotent $r$-subgroup.

This proves the second part of Theorem 4.1.

\section{Acknowledgments}

The authors would like to thank Greg Cherlin and Tuna Altınel for their helpful comments and advice.

\section{References}

[AB08] Tuna Altmel and Jeffrey Burdges. On analogies between algebraic groups and groups of finite Morley rank. Submitted, 2008.

[ABC07] Tuna Altınel, Alexandre Borovik, and Gregory Cherlin. Simple groups of finite Morley rank. Book in preperation, 2007.

[ABCC01] Tuna Altmel, Alexandre Borovik, Gregory Cherlin, and Luis-Jaime Corredor. Parabolic 2-local subgroups in groups of finite Morley rank of even type. Preprint, 2001. 
[BB04] Ayşe Berkman and Alexandre V. Borovik. A generic identification theorem for groups of finite Morley rank. J. London Math. Soc. (2), 69(1):14-26, 2004.

[BBC07] Alexandre Borovik, Jeffrey Burdges, and Gregory Cherlin. Involutions in groups of finite Morley rank of degenerate type. Selecta, 13(1):1-22, 2007.

[BC08] Jeffrey Burdges and Gregory Cherlin. Semisimple torsion in groups of finite morley rank. Submitted, 2008.

[BCJ07] Jeffrey Burdges, Gregory Cherlin, and Eric Jaligot. Minimal connected simple groups of finite Morley rank with strongly embedded subgroups. $J$. Algebra, 314(2):581-612, 2007.

[Ber01] Ayşe Berkman. The classical involution theorem for groups of finite Morley rank. J. Algebra, 243(2):361-384, 2001.

[BN94] Alexandre Borovik and Ali Nesin. Groups of Finite Morley Rank. The Clarendon Press Oxford University Press, New York, 1994. Oxford Science Publications.

[Bur04] Jeffrey Burdges. A signalizer functor theorem for groups of finite Morley rank. J. Algebra, 274(1):215-229, 2004.

[Bur07] Jeffrey Burdges. The Bender method in groups of finite Morley rank. J. Algebra, 307(2):704-726, 2007.

[Che05] Gregory Cherlin. Good tori in groups of finite Morley rank. J. Group Theory, 8:613-621, 2005.

[Del07] Adrien Deloro. Groupes simples connexes minimaux de type impair. PhD thesis, Université Paris 7, Paris, 2007.

[Fré08] Olivier Frécon. Conjugacy of carter subgroups in groups of finite Morley rank. J. Math Logic, 2008. Submitted.

[Gor80] Daniel Gorenstein. Finite groups. Chelsea Publishing Co., New York, second edition, 1980 .

[HW] Ehud Hrushovski and Frank Wagner. Counting and dimensions. To appear.

[Wag97] Frank O. Wagner. Stable groups. Cambridge University Press, Cambridge, 1997. 